\title{
Hacia una nueva mirada de la reintegración de desmovilizados en Colombia: conceptos, enfoques y posibilidades ${ }^{* *}$
}

Towards a new look at the reintegration of demobilized persons in Colombia: concepts, approaches and possibilities

Rumo a uma nova visão sobre a reintegração de desmobilizados na Colômbia: conceitos, abordagens e possibilidades

\footnotetext{
* Estudiante de Maestría en Estudios Sociales y Políticos, docente e investigador en formación, y sociólogo y profesional en mercado internacional y publicidad de la Universidad Icesi. Correo electrónico: jdmesa@icesi.edu.co

${ }^{* *}$ Este artículo se enmarca en uno de los objetivos centrales de la investigación Reintegración laboraly vida cotidiana en Cali para optar por el título de Magister en Estudios Sociales y Políticos de la Universidad Icesi. Artículo de investigación recibido el 07-06-2017 y aprobado 21-07-2017.
} 


\section{Cómo citar}

MESA, J. D. (2017). Hacia una nueva mirada de la reintegración de desmovilizados en Colombia: conceptos, enfoques y posibilidades. Revista CS, 23, pp. 105-133.

DOI: http://dx.doi.org/10.18046/recs.i23.2437 
Este artículo pone en evidencia que la agenda de la política pública de la reintegración en Colombia ha estado estrechamente articulada al mandato internacional del programa de Desarme, Desmovilización y Reintegración, que asume el proceso de forma homogénea, sin considerar los aspectos singulares de los reintegrados (su grupo de procedencia, género, raza, entre otros). Se señala, así mismo, que la agenda académica de la reintegración en Colombia, que se ha encargado de diagnosticar lo anterior, se encuentra todavía en una fase de discusión jurídico-política, pues se ha concentrado en los marcos jurídicos, estudios institucionalistas y comparativos del proceso, desde una apuesta teórica fundamentalmente estructural-funcionalista. En esa medida, se propone una mirada teórico-metodológica alternativa, desde el pragmatismo de la teoría de la acción creativa de Hans Joas, partiendo de los aspectos positivos y la reformulación de los más problemáticos del estado de la cuestión.

PALABRAS CLAVE:

DDR | Colombia | Reintegración | Teoría de la acción creativa

This article shows that the public policy agenda of reintegration in Colombia has been closely linked to the international mandate of the Disarmament, Demobilization and Reintegration program, which takes the process in a homogeneous way without considering the unique aspects of demobilized persons (their group of origin, gender, race, among others). It is also pointed out that the academic agenda of reintegration in Colombia, which has been in charge of diagnosing the above, is still in a phase of legal-political discussion because it has concentrated on the legal frameworks, institutionalist and comparative studies of the Process, from a fundamentally structural-functionalist theoretical bet. To that extent, an alternative theoretical-methodological approach is proposed, from the pragmatism of Hans Joas's theory of creative action, starting from the positive aspects and the reformulation of the most problematic of the state of the matter.

KEYWORDS:

DDR | Colombia | Reintegration | Theory of creative action 
Neste artigo se evidencia que a agenda da política pública da reintegração na Colômbia tem estado estreitamente articulada ao mandato internacional do programa de Desarmamento, Desmobilização e Reintegração que assume o processo de forma homogênea sem considerar os aspectos singulares dos reintegrados (seu grupo de procedência, gênero, raça, entre outros). Assinala-se também que a agenda acadêmica da reintegração na Colômbia, que tem se encarregado de diagnosticar o que está acima mencionado, encontra-se ainda em uma fase de discussão jurídico-política, pois tem se concentrado nos marcos jurídicos, estudos institucionalistas e comparativos do processo, a partir de uma aposta teórica fundamentalmente estrutural-funcionalista. Nessa medida, propõe-se uma mirada teórica-metodológica alternativa, desde o pragmatismo da teoria da ação criativa de Hans Joas, partindo dos aspectos positivos e a reformulação dos mais problemáticos do estado da questão.

PALAVRAS CHAVES:

DDR | Colômbia | Reintegração | Teoria da ação criativa 


\section{Introducción}

La reintegración se constituye en uno de los tres pilares de los procesos de disgregación de los grupos armados de la clandestinidad y de la ilegalidad, siendo los otros dos pilares de este proyecto institucionalizado, en tanto política de Estado con mandato global, el desarme y la desmovilización. Así, el programa de Desarme, Desmovilización y Reintegración -DDR-, que sintetiza la apuesta cronológica del proceso, es un acuerdo con alcance global porque se inscribe en las decisiones de los países que hacen parte de la ONU para hacer frente a los conflictos internos. De modo que, culminados el desarme y la desmovilización con la vigilancia de dicha entidad internacional, la reintegración aparece como la etapa más amplia del proceso, en tanto busca la transición hacia la vida civil y legal de los desmovilizados a partir de un acompañamiento estatal constante en diversas esferas de la vida cotidiana de estos sujetos de interés. En Colombia, específicamente, la reintegración la constituyen ocho dimensiones de intervención que lleva a cabo la Agencia Colombiana para la Reintegración -ACR ${ }^{1-}$ en lo que se denomina la «Ruta»: las dimensiones personal-psicosocial, familiar, comunitaria, en salud, educativa, ciudadana, en seguridad y productiva.

Cada dimensión refleja un interés puntual con respecto a la formación de ciudadanos, en términos de un ideal normalizado socialmente y apropiado institucionalmente. Por esto, además de recibir atención psicosocial de forma transversal en el proceso, el excombatiente en proceso de reintegración debe acoplarse a la vida familiar, comunitaria, urbana o rural (según sea el proceso) para tener un entorno seguro; además debe acceder a programas de salud y educación para sensibilizarse frente a aquellas técnicas y conocimientos que le pueden permitir hacer frente a la vida cotidiana en el contexto de la legalidad: ser un ciudadano saludable según las expectativas ciudadanas y tener conocimientos de ciertas técnicas y tecnologías para que su reintegración laboral sea exitosa. De ahí que la dimensión laboral, específicamente, llamada «esfera productiva», en la Ruta de la ACR, sea neurálgica para culminar exitosamente el proceso y evitar la «reincidencia» de estos sujetos en la vida armada. La reintegración laboral se constituye a partir de dos procesos: empleabilidad y emprendimiento (ACR, 2016).

Pero, ¿cómo afronta el desmovilizado, cotidianamente, la reintegración? Más allá de la mirada institucional, es clave pensar el proceso también desde quien lo

1. El 29 de mayo de 2017, a partir del decreto 897, pasó a llamarse Agencia para la Reincorporación y Normalización-ARN-. En este artículo, sin embargo, se denomina a la agencia a partir de su nomenclatura anterior, ACR, teniendo en cuenta que la agenda de la política pública y de la academia, a la que se hace referencia, se basó en el primer acrónimo. 
asume (o no) en su propia experiencia, y no solo desde la estructura que lo propone: porque el agente, en su interpretación del proceso, también estructura la estructura, si consideramos la propuesta bourdieusiana. En ese sentido, mi interés en este artículo radica fundamentalmente en poner en diálogo y evidencia los trabajos más importantes sobre la reintegración: ¿qué se ha investigado en el campo del Desarme, la Desmovilización y la Reintegración-DDR-en Colombia? ¿Específicamente, qué hay acerca de las dimensiones de la reintegración? ¿Cómo se ha investigado? En resumidas cuentas, ¿cuáles han sido los conceptos o enfoques teóricos y metodológicos desde donde se ha abordado el tema de la reintegración? ¿Y qué aspectos, dimensiones teóricas o enfoques metodológicos son positivos, problemáticos y valdría la pena reformular? ¿Cómo reformularlos?

En este artículo abordaré, en primer lugar, teórica y metodológicamente, trabajos acerca de DDR en Colombia, teniendo en cuenta procesos contemporáneos y antecedentes históricos que devengan de los diversos desmovilizados de los múltiples grupos armados que ha tenido el país. En segundo lugar, sintetizaré los conceptos o enfoques teóricos y metodológicos sobre la reintegración para sustraer los aspectos positivos y resaltar aquellos problemáticos que valdría la pena reformular. En tercery último lugar, propondré una mirada alternativa partiendo de los aspectos positivos y la reformulación de los problemáticos desde el pragmatismo de la teoría de la acción creativa de Hans Joas (2013) para ampliar el campo de posibilidades en este tema.

\section{Revisión de la agenda de la reintegración}

\section{Conceptos}

Uno de los trabajos más significativos en el país sobre DDR en Colombia es la propuesta de Labrador y Gómez (2010) en su libro titulado Desarme, Desmovilización y Reintegración en Colombia. En este libro los autores hacen una minuciosa revisión documental de instrumentos institucionales, como el CONPES 3554 de 2008, entre otros documentos de carácter jurídico, para proponer una definición vigente de las categorías del DDR: Desarme, Desmovilización y Reintegración. Esto es, desarmado como quien tuvo la voluntad política de entregar las armas; desmovilizado como quien se diluye y deja la unidad armada y sus funciones respectivas con intención de retornar a la vida civil y legal; reintegrado como quien atravesó exitosamente el proceso de recobrar su carácter civil de forma sostenible, legal y sin violencia (Labrador y Gómez, 2010). 
A partir de estas definiciones, surgen dos cuestiones conceptuales por revisar en la literatura, a propósito de este tema. Por un lado, aclarar las diferencias conceptuales entre dos categorías aparentemente iguales pero, según varios autores, dísimiles: reinserción y reintegración. Por otro lado, reflexionar en torno a qué tan válido es referirse, de forma universal, a re-integración para casos de desmovilizados que no han vivido, en carne y hueso, en la legalidad (casos, por ejemplo, de niños en la guerrilla): pues la re-integración así pensada supondría un proceso en donde se les estaría posibilitando a estos sujetos de interés volver a una fase anterior que ya vivieron, la de la vida civil. O es que acaso, ¿todos nacemos siendo legales per se?

Años antes, con respecto al concepto de reinserción, Correa (2007) ofrecía una serie de pistas teóricas. Esta propuesta es interesante para comprender, en términos jurídicos, qué es la reinserción y cuál es su relación con la categoría reparación en el marco de la Ley 975 de 2005 sobre unidad de víctimas. El argumento de fondo de Correa es que la reinserción debe estar sujeta, concomitantemente, al proceso de reparación de las víctimas, por cuanto esto asegura que el desmovilizado comience a reconocer un marco de la legalidad al que se debe someter para integrarse a la vida civil; esto es, como garantía de la no repetición. Esta perspectiva enriquecerá el marco de referencia para un escenario en donde sea clave re-pensar la categoría genérica de reintegración en dos esferas: en términos normativos y legales, y en su relación, aparentemente dialéctica, con la reparación de las víctimas del conflicto.

Pero entonces, ¿cuál es la diferencia entre reinserción y reintegración? Para comprender esta diferencia es importante primero hacer una revisión de ambos conceptos a profundidad. Pérez (2005) también hace una juiciosa revisión de la Ley 975 de 2005, disponible hasta ese momento en Colombia, buscando rastrear el concepto de reinserción: ¿qué dicen las leyes sobre este fenómeno? Su argumento radica en pensar cómo la reinserción implicó una reconfiguración del orden jurídico en Colombia. Es decir, cómo al empezar a hablar de reinserción se tuvo que ajustar el discurso inmiscuido en las normas en su carácter vinculante: del discurso normativo para hacer frente (solo) a la guerra, al derecho para gestionar la convivencia. De ese modo, vale rescatar dos asuntos importantes que se abordan en esta propuesta. Por un lado, pensar la coyuntura histórica -desde la reintegración, pero en general desde los postacuerdos mismos- como fundadores de órdenes jurídicos flexibles y adaptativos. Por otro lado, profundizar más con respecto al abordaje y revisión metodológica de las leyes y los marcos jurídicos que hace el autor, para rastrear, con leyes más actuales, el concepto.

Tal vez Giraldo (2010) es de las pocas autoras que recurre a diferentes conceptualizaciones para estudiar la reintegración a partir de sus antecedentes históricos, de políticas públicas y programas dirigidos hacia su gestión y administración en 
Colombia, comparando cómo fue y cómo ha sido su implementación en términos del deber ser del proceso, inscrito en el discurso institucional. La autora no solo se limita a revisar la documentación jurídica disponible sino que además recurre a diferentes actores implicados, sobre todo del lado de la implementación: funcionarios públicos. Su propuesta fue el resultado de la primera parte de una investigación titulada: Análisis de la política pública de reintegración social y económica en Bogotá durante 2006-2009. Es por esto que sus reflexiones están delimitadas en dicho periodo, aunque retome, como referencia cronológica anterior, los procesos de reintegración que se comenzaron a desarrollar a partir de 2002: buscando contrastar la política de «seguridad democrática» implantada por el entrante presidente Uribe con las desmovilizaciones suscitadas a partir de ese momento.

De ese modo, Giraldo (2010) problematiza la reintegración en Colombia, justificando que ante la falta de antecedentes por la ausencia de programas formales y políticas públicas con ese objetivo antes de 2002, en los dos gobiernos de Uribe, también considerando su ideología de la seguridad democrática, se plantearía una dinámica de gestión de la desmovilización desde el discurso mismo del conflicto, y no desde la reconciliación: como se establece en muchos países con sus políticas de DDR (Labrador y Gómez, 2010). En los procesos anteriores a 2002, dice la autora, se beneficiaron solo aquellos guerrilleros que contaban con un liderazgo político para ocupar un lugar en la vida pública: como en el caso de la desmovilización colectiva del M-19. Además, el proceso, informal antes que nada, presentaba modificaciones según la conveniencia política de cada gobierno.

Sumada la informalidad y poca experiencia que había en procesos de reintegración en Colombia, con la política de seguridad democrática de Uribe, la gestión de la desmovilización entre 2002 y 2010 sería, para la autora, ineficiente; esto es, un apéndice más de la guerra que no se separó del conflicto: desmovilizado, el ex guerrillero seguía teniendo un estatus de guerrillero. Con esto en mente, la autora plantea como hipótesis que la continuidad en las condiciones que generan y reproducen el conflicto armado colombiano y el manejo institucional que se le dio entre 2002 y 2010, antes de la creación de la ACR, no permitieron en ese momento llevar a cabo un proceso de reintegración efectivo. De esta crítica se despliegan cinco argumentos de parte de la autora para indicar las causas para la poca efectividad del proceso de reintegración en Colombia en el periodo mencionado.

En primer lugar, según la autora, la fuerte desigualdad en el acceso a un espacio vital mínimo (salud, educación, vivienda y trabajo) de parte de los desmovilizados, implicó, paradójicamente, que se mantuvieran, en el proceso mismo de reintegración, las condiciones de la existencia del conflicto armado y desigualdad en 
Colombia: condiciones por las que muchos de los desmovilizados que comenzaron su proceso de reintegración, alguna vez opusieron como argumento para la lucha armada. Según la autora, en muchos casos, incluso, en donde no había certidumbre de parte de los desmovilizados con respecto a su acceso a estos mínimos vitales, hubo reincidencia al grupo armado. La contradicción y paradoja de fondo es política: ¿cómo reintegrar efectivamente a aquellos desmovilizados que por su discurso político buscan que se les reivindique con aquello por lo que justifican su lucha armada, cuando esa reivindicación no está asegurada?

En segundo lugar, los programas de reintegración presentaron múltiples fallas estructurales, debido a la limitación de recursos humanos y presupuestales, «que desbordaba su capacidad para atender a 1.20o desmovilizados individuales y 31.687 colectivos» durante dicho periodo (Giraldo, 2010: 39). En tercer lugar, hubo una fuerte ausencia de coordinación institucional por la multiplicidad de organizaciones involucradas en el proceso de reintegración. La autora menciona la intermediación de diferentes programas y oficinas que burocratizaron y generaron duplicidad de funciones en la política pública: Programa de atención humanitaria al desmovilizado del Ministerio de Defensa (PAHD), Oficina del Alto Comisionado para la Paz, Programa para la reincorporación a la vida civil de ex combatientes y alzados en armas (PRVC), entre otros.

Siguiendo la línea del anterior argumento, en cuarto lugar, Giraldo (2010) menciona que los programas presentaron una fuerte desarticulación entre el gobierno nacional y los gobiernos locales. La responsabilidad en la reintegración recaía, fundamentalmente, en los gobiernos locales, a pesar de que los programas habían sido planteados por el gobierno nacional sin pensar, irónicamente, en la participación de los primeros; como aparece en el Decreto 200 de 2003. El último argumento que plantea la autora, ligado al primero ya mencionado, es que hubo tratamiento diferencial de los desmovilizados en el acceso a la asistencia humanitaria que proponía el PAHD y el PRVC. Para poner un ejemplo, mientras que algunos desmovilizados recibieron colectivamente 2 millones de pesos de parte de la Oficina del Alto Comisionado para la Paz, los individuales obtuvieron hasta 8 millones del PRVC (Giraldo, 2010: 40).

Con respecto a la diferencia conceptual planteada antes como interrogante, debido a las problemáticas que esto suscitó, la autora señala que hacia finales del 2006, reforzándose en 2010, se planteó una estrategia con un enfoque diferente al que se venía presentando. Esa estrategia incluyó, como punto de partida, un cambio semántico de la comprensión del proceso DDR. Así, la «R» de Reinserción o Reincorporación que se veía manejando, y que implicaba discursivamente un proceso específico que nunca logró despegarse del conflicto, se reemplazó por la «R» de Reintegración: que 
a su vez comenzó a conjugarse mejor con la «R» de Reconciliación. Estas categorías, junto con el Desarme y la Desmovilización, se convirtieron entonces en variables centrales para comprender, desde un marco teórico breve y claro que propone Giraldo (2010), el proceso del (ideal) DDR: que debe estar debidamente acompañado, claro está, por las instituciones para así evitar errores pasados.

Pero, desde la mirada global de la ONU, institución instauradora del proceso, y otras autorías, ¿qué se concibe como DDR? El Desarme, según la ONU (2006), es el proceso de recolección, control y disposición de todo tipo de armas que estén en poder de los combatientes. La Desmovilización, por otro lado, supone cinco pasos fundamentales: planificación, campamento, registro, orientación antes de la desmovilización y licenciamiento absoluto de los ex combatientes. La Desmovilización es sumamente importante porque simboliza el cambio de una vida militar a una civil: es una ruptura con el comando y la posibilidad de fracciones armadas, de modo que supone el primer paso para la Reintegración. Transversalmente, la apuesta de este programa, desde esa agenda global, es la construcción de Estado y escenarios de paz.

Esto es visible, por ejemplo, en los casos centroamericanos de DDR (Nicaragua, El Salvador y Guatemala), en donde los estudios en este campo se han focalizado en las reflexiones en torno a retrospectivas críticas del proceso, esto es, principales aprendizajes, errores, alcances, etc. El enfoque principal que subyace de estos estudios se concentra en una preocupación por la reintegración en el espectro político: la construcción de Estado y escenarios de paz considerando los nuevos actores en pugna por el poder (Chamorro, 2015; Escuela de Cultura de Paz, 2011). En el caso de Nigeria, ocurrió algo similar con el grupo alzado en armas «Niger-Delta», algunos autores han investigado los marcos jurídicos-políticos en los que se inscribieron los acuerdos de amnistía a sus integrantes, con una discusión intrínseca, de nuevo, por la construcción de Estado y paz en contextos violentos (Ajayi y Adesote, 2013; Courson, 2011; Davidheiser y Nyiayaana, 2011).

Otro caso país esencial en donde se evidencia esta apuesta es el de la desmovilización en la República Democrática del Congo. Zirion (2012) incluye la variable género cuando analiza cómo fue la participación de las mujeres en los procesos de desarme, desmovilización y reintegración de excombatientes y en la construcción de paz y Estado en este país. Argumenta que, dado que no se generó una participación por parte de las mujeres en este proceso, por no formar parte de la agenda de paz, su proceso de reintegración estuvo lleno de obstáculos, si se compara con el de los hombres que sí hicieron parte activa del DDR. Este asunto ayuda a contemplar un interrogante neurálgico a propósito de la reintegración en Colombia: ¿tendrá implicaciones en el proceso de reintegración el género de el o la desmovilizada, teniendo en cuenta que Colombia está marcada por una fuerte desigualdad entre 
las oportunidades (laborales, educativas, etc.) de las mujeres frente a los hombres? Como ya veremos, este aspecto singular es neurálgico, aunque en ninguna de las dos agendas (la institucional y la académica) exista explícitamente.

Ahora bien, siguiendo en la revisión, Gleichman et al. (2004) proponen una guía teórica sobre la temática, sostienen que la reinserción -para no confundirla con la reintegración- es el procedimiento inmediatamente anterior a esta. Implica, en ese sentido, el traslado de los combatientes a los municipios y regiones de su preferencia. Ya instalados los desmovilizados, la reinserción incluye el abastecimiento de alimentos, ropa, asistencia médica, dinero, herramientas de trabajo y alojamiento incluido, para estos ex combatientes y sus respectivas familias. En otras palabras: «La reinserción se refiere al período breve de la llegada del ex combatiente a su antigua residencia o a una nueva comunidad» (Anderlini y Conaway, 2006: 2).

La reintegración, como etapa final del DDR, es el proceso en el cual «los excombatientes adquieren estado civil y tienen acceso a formas civiles de trabajo e ingresos. Esencialmente, es un proceso social y económico dentro de un período de tiempo indeterminado que tiene lugar principalmente en las comunidades a nivel local» (Gleichman et al., 2004:4). Es decir, que la reintegración debe garantizar que los desmovilizados no retornen a la violencia, también incentivar la construcción de vínculos de confianza y tolerancia con las comunidades urbanas o rurales donde viven, trabajan, estudian, etc. Por esto es clave que la reintegración se articule con procesos visibles de reconciliación: de ahí que Giraldo (2010) sostenga que la reintegración es un proceso que incluye activamente a la sociedad civil. La reconciliación, de esta manera, es un proceso, en el contexto de construcción de la paz, en donde se procura una transformación de las emociones ligadas al sufrimiento del pasado, dando paso a relaciones constructivas y tolerantes. Como lo indican Humphreys y Weinstein (2007), la reconciliación es fundamental para el DDR en su conjunto porque tanto los desmovilizados como sus familias precisan de la aceptación de las comunidades de acogida, pues la construcción de paz inicia con estos actos cotidianos simbólicos del día a día.

\section{Enfoques}

Con respecto a los enfoques, las propuestas se han concentrado en los marcos jurídicos, estudios institucionalistas y comparativos del proceso. Carranza (2012), por ejemplo, plantea el papel que han jugado las administraciones municipales en Colombia en el proceso de reintegración. De ese modo, se pregunta cómo se han articulado o no las políticas de la ACR, desde su nacimiento en 2011, con los proyectos especiales planteados desde las Alcaldías de Bogotá y Medellín, en sus Secretarías o 
Direcciones administrativas. A partir de este interrogante, a través de una revisión de los proyectos y una serie de entrevistas a funcionarios públicos, la autora sostiene que mientras no haya una integración clara de políticas y programas, como ha ocurrido en algunos casos, se va a generar una dinámica de duplicidad de medidas estatales que desaceleran y burocratizan el proceso de reintegración. Giraldo y Mesa (2013), de forma similar, analizan el papel de los entes gubernamentales del municipio de Medellín, desde mediados de los 9o, en el proceso DDR de las milicias urbanas de grupos paramilitares. Su propuesta, devengada a través de un análisis de datos cuantitativos y de los programas del municipio, es que se llevaron a cabo procesos de reintegración sin que los grupos de milicianos se desmovilizaran de las estructuras armadas y sus prácticas, pues continuaron incidiendo fuertemente en el control territorial, el microtráfico y el terror, a la vez que recibían los beneficios del programa estatal, como fue el caso de La Sierra en la comuna 8 de Medellín (Mesa, 2015).

Comprendiendo este marco conceptual, que ha sido objeto de estudio en esas investigaciones, Villarraga (2013) propone cómo han sido las diferentes experiencias históricas de reintegración en Colombia con los distintos grupos armados (M-19, EPL, FARC, ELN, AUC). Su propuesta, que parte también de una revisión histórica de la documentación institucional relacionada con el tema, es que el proceso de reintegración, desde los años 90 y con la creación de la Agencia Colombiana para la Reintegración (ACR) con el Decreto 4138 de 2011, se ha pensado y aplicado de forma homogénea en todos los desmovilizados de los diferentes grupos armados. Con una propuesta similar -en dos informes sobre el proceso de desmovilización de las AUC para el Centro Nacional de Memoria Histórica - Villarraga (2015a y 2015b) hace una evaluación crítica de la posdesmovilización de este grupo en donde además plantea cómo las dificultades en el DDR y la Ley de Justicia y Paz, entre otras razones, llevaron a que muchos de los ex combatientes se rearmaran y formaran las denominadas «bandas criminales».

Esto, según el autor, ha complicado el proceso, entre otras cosas, porque no se han considerado las singularidades propias de cada actor armado a la hora de reintegrarlos a la vida civil: ¿implica lo mismo reintegrar a un desmovilizado del ELN y que a uno de la FARC?2 ¿Implica lo mismo reintegrar a un desmovilizado de una guerrilla que a uno de un grupo paramilitar? ¿Implica lo mismo reintegrar a un desmovilizado que a una desmovilizada? ¿Implica lo mismo reintegrar a un desmovilizado afro que a un desmovilizado mestizo? Lo curioso del asunto es que la mayoría de investigaciones sobre el tema tampoco han considerado dichas singularidades

2. En singular considerando su nueva denominación como partido político: Fuerza Alternativa Revolucionaria del Común. 
porque metodológicamente ni siquiera las han abordado: la agencia del sujeto de interés, en términos de su género, raza, grupo de procedencia, etc., ha sido relegada.

Una de las excepciones a esto se encuentra en la propuesta reciente de Hundek (2016) que llama la atención sobre la importancia de reconocer que la reintegración se asume de forma diferencial en hombres y mujeres. En sus palabras sostiene que «si la reinserción a la vida civil fue un proceso traumático para los combatientes en general, para la mujer reinsertada lo fue mucho más si se reconoce la prevalencia de un contexto socio-cultural que mantiene la inequidad de las relaciones de género» (Hundek, 2016: 65). La dificultad reside en que las mujeres provienen de estructuras armadas en donde las diferencias de género no son tan marcadas (como es el caso de la FARC) y llegan a la vida civil, en donde la estructura patriarcal las limita a roles domésticos, sexuales y reproductivos. En esta propuesta no hay, sin embargo, un relato sobre la resistencia cotidiana de estas mujeres a esa realidad adversa: se privilegia el peso de la estructura sobre los intereses de las agentes.

Otra de las excepciones es el informe Centro Nacional de Memoria Histórica, CNMH (2014) Desafios para la reintegración: enfoques de género, edady etnia. A través de un ejercicio de investigación exhaustivo y minucioso de la reintegración en el país, este informe es fundamental porque propone un enfoque diferencial en el proceso de reintegración. Esto, precisamente, porque se parte del hecho de que el conflicto armado ha traído un impacto desproporcionado en los distintos actores, no solo por su condición de víctima o victimario sino también porque la violencia ha golpeado de formas distintas a las poblaciones según su condición étnica y de género. Es por eso que se propone una mirada con una perspectiva interseccional que aborde los procesos de reintegración según la identidad de género, la orientación sexual y la edad de la persona implicada. Cabe agregar que este informe es valioso porque considera la experiencia de múltiples ex combatientes mujeres, de sectores LGBT, e infantes en sus procesos de reintegración, aunque relega un poco de la discusión el asunto étnico.

Haciendo referencia, concretamente, a las dimensiones de la reintegración (personal-psicosocial, familiar, comunitaria, en salud, educativa, ciudadana, en seguridad y productiva), el campo investigativo en Colombia ofrece un panorama desolador al respecto. Gómez (2007), por ejemplo, es de las pocas autoras que reflexiona sobre la relación entre las transformaciones en el mercado de trabajo y el proceso de paz en Colombia para explicar la dimensión productiva de la reintegración. En ese sentido, sostiene que las condiciones laborales mismas, dadas en un contexto normativo del mercado laboral en Colombia, pueden obstaculizar la reintegración de los desmovilizados a la esfera social. Pero, de nuevo, su propuesta se limita a una revisión de la documentación institucional respectiva. De forma particular, afirma cómo, por ejemplo, los trabajos indirectos, que suponen menos estabilidad laboral, 
implican grandes dificultades en la reintegración laboral de los desmovilizados: pero la voz de los implicados no aparece explícitamente.

Romero et al. (2009) proponen los factores psicosociales que han incidido en la reintegración social de integrantes de grupos armados ilegales (FARC, UC-ELN y AUC) que se encuentran recluidos en cárceles de Cali. Partiendo de una metodología cualitativa concentrada en tres trayectorias puntuales, estas autoras encontraron que la percepción que tienen estos ex combatientes sobre el conflicto armado articula elementos estructurales, económicos y culturales que en su discurso legitiman su reproducción. Además, encontraron que la dinámica del grupo armado ilegal se replica en la dinámica grupal en la prisión: lo que dificulta el proceso de reintegración considerando el factor adicional de exclusión por ser población carcelaria. Tamayo et al.(2012) hacen un diagnóstico del proceso de educación en salud que han recibido los desmovilizados de diversos grupos armados en Medellín. A través de una metodología cualitativa concentrada en entrevistas a profundidad, sobre todo a funcionarios de salud, estas autoras encontraron que dicho proceso de educación en salud se ve condicionado necesariamente a la afiliación al Sistema General de Seguridad Social en Salud, y al apoyo que la EPS e IPS puedan ofrecer al desmovilizado. En cuanto al análisis de las dimensiones de la reintegración, en las pocas investigaciones realizadas sobre los diversos temas, la mirada se ha concentrado en la institución (Estado, ACR, etc.) como la que determina el éxito o fracaso del proceso.

Es visible, entonces, la predominancia de una agenda estructural-funcionalista en el proceso de reintegración: en donde, en la mayoría de investigaciones, la voz de los implicados no parece ser relevante. Esto da cuenta de una suerte de «inocencia epistemológica», tomando prestada la expresión de Bourdieu (2008), porque en muchos estudios se afirma comprender el proceso de la reintegración solo abordando la coyuntura institucionalista, sin incorporar la voz y la experiencia de los implicados directamente en las investigaciones.

Cabe señalar, sin embargo, dos propuestas que superan dicha agenda, aunque sigan presentando una mirada de estos sujetos como una construcción sometida necesariamente al colectivo. Luna (2007), por ejemplo, aborda la dimensión subjetiva de los ex militantes del M-19 que, en su momento, no atravesaron en sí un proceso de reintegración porque no existía en la época una política del DDR, como ya lo he señalado. De todos modos esta investigación es destacable porque el autor recurre a la historia de vida a partir de la recolección de narrativas desde la entrevista, lo que convierte a esta propuesta en una de las pocas en reconocer de «viva voz» a los implicados directamente en el proceso. Luna (2007) propone que es necesario dejar de asumir que la acción de los ex militantes de dicho grupo solo responde a 
los intereses y discursos de la colectividad. Más allá de esa mirada omnipresente de la acción colectiva, estos sujetos tienen un reconocimiento subjetivo de sí mismos que trasciende las perspectivas colectivistas del grupo al que pertenecieron. En sus múltiples esferas cotidianas, el desmovilizado también actúa con base en sus propios intereses.

Nussio (2012), de forma similar, se concentra en la vida después de la desmovilización de un grupo de ex paramilitares en tres zonas geográficas distintas: Bogotá, Antioquia y Santander. A partir de entrevistas en profundidad a 54 reintegrados y reintegradas, y desde una perspectiva de género, el autor narra cómo los implicados apelaron a diversas estrategias cotidianas para sobrellevar los estigmas de su vida pasada ante diversos escenarios y situaciones adversas que se presentaban en el proceso. En esa medida, Nussio (2012 y 2013) describe las contradicciones, antagonismos y peculiaridades que implicó para dichos reintegrados el programa de reintegración, lo que deja entrever el papel estructurante de la agencia: por ejemplo, no seguir a cabalidad los consejos de la «Ruta» de la reintegración y apelar a estrategias que consideraran más efectivas de acuerdo a la situación.

En el caso de las mujeres, es interesante resaltar el siguiente hallazgo: el autor encontró que para las reintegradas era más fácil reintegrarse comunitariamente que laboralmente, pues sus estigmas se concentraban en sus supuestas incapacidades para ejercer una labor por fuera del ámbito doméstico. En el caso de los hombres, es interesante resaltar el siguiente hallazgo: el autor encontró que para los reintegrados era más fácil reintegrarse laboralmente que comunitariamente, pues sus estigmas se concentraban en sus supuestas incapacidades para generar confianza en los demás. Dichos hallazgos denotan la importancia de asumir la perspectiva de género en la agencia, así como el papel de la raza en la construcción del estigma, por ejemplo.

Como en el caso de la propuesta de Luna (2007), en Nussio (2012) es destacable el enfoque metodológico que se corresponde con su apuesta teórica más centrada en la agencia y el interaccionismo propuesto desde Goffman (2004). Igualmente, es destacable un hecho irrebatible: este estudio es de los pocos en Colombia que aborda la desmovilización desde la perspectiva de ex paramilitares, contando los informes de Villarraga (2015a y 2015b). De modo que, además de que hay pocas investigaciones que consideran los aspectos singulares de los desmovilizados en el proceso de reintegración, las investigaciones en este campo no han abordado las múltiples dimensiones que implica. 


\section{Hacia una nueva mirada: la importancia de la agencia del reintegrado}

¿Qué mirada teórica-metodológica sería más apropiada para comprender la reintegración en Colombia? ¿A partir de qué conceptos? ¿Qué posibilidades ofrecería otra mirada? De antemano, quiero dejar claro un asunto: no hay una sola forma de abordar un tema; hay múltiples opciones, paradigmas y enfoques. Sin embargo, hay aspectos desde lo teórico y lo metodológico, según la particularidad de un tema, que difícilmente son negociables y que merece la pena llevar a cabo. Es por esto que en esta sección me interesa proponer una mirada teórica-metodológica alternativa de la reintegración partiendo de los aspectos relevantes y la reformulación de los más problemáticos del estado de la cuestión señalados anteriormente. Lo fundamental de mi propuesta es superar la «inocencia epistemológica» de la agenda estructural-funcionalista de la literatura sobre reintegración en Colombia, dejando clara la importancia de la agencia para legitimar teórica y metodológicamente la comprensión de la reintegración.

Mi argumento es que no se puede comprender la reintegración sin considerar al directamente implicado: el reintegrado mismo y su agencia sobre lo institucional. Se trata de reconocer, como lo proponen algunas investigaciones del tema (Luna, 2007; Nussio, 2012), que el reintegrado no solo está determinado por la institución: el reintegrado en sí, en su interpretación cotidiana del programa de reintegración, también reconfigura ese orden institucional establecido, esto es, genera cambio social. En esa medida, propongo un enfoque pragmatista desde la teoría de la acción creativa de Joas (2013) en donde se reconozca no solo la importancia de los aspectos institucionales (los marcos jurídicos, por ejemplo) sino también la agencia de los reintegrados para resolver problemas cotidianos-situados sin necesariamente responder al dictamen estructural de la institución. Para que la reflexión esté completa, propongo además considerar de forma transversal el concepto de habitus en Bourdieu (2007), para abordar teórica y metodológicamente la reintegración. Así mismo, retomo la propuesta de Vergara (2014) sobre cuerpo vaciado y estigma, como ejemplo para reconocer la importancia de la raza para pensar un asunto como la reintegración. Lo propio haré con Farnsworth-Alvear (2000) con respecto a su forma de incluir el género, y su interesante propuesta metodológica que puede ser clave para pensar la reintegración como problema de investigación.

Para empezar, es importante señalar que en el campo de los estudios sobre agencia hay figuras académicas neurálgicas, como Scott (1976 y 2008) y Gell (1998), por mencionar dos de las más relevantes. Estas propuestas, sin embargo, no asumen explícitamente el papel del cuerpo como unidad ineludible de la agencia y no le 
dan lugar a la singularidad situacional: la instancia del subjetivismo supera a la del objetivismo en estos casos. Scott (1976 y 2008), retomando la economía moral de la multitud de Thompson (2000 y 2014), propone la categoría de resistencia como entidad propia de la subalternidad para oponerse a la dominación. ${ }^{3}$ Esa resistencia es comunicada, compartida y representada por parte del grupo subalterno como un ethos en sí mismo, precisamente porque la dominación es un aspecto compartido. En este contexto, la agencia, en tanto resistencia, surge como respuesta a la estructura de dominación que es vivida de la misma forma por parte de los implicados: como una respuesta contra-hegemónica. En esta propuesta, pues, no hay agencia por fuera del ethos, por fuera de la explotación, lo que implica que la vida cotidiana es en sí misma un escenario de dominación las 24 horas del día: lo que no es extrapolable a un contexto urbano como el de la reintegración en Colombia, en donde si bien prima el estigma, este está ligado a la situación.

Gell (1998), por otro lado, propone la categoría de animismo vehicular para señalar que los objetos tienen agencia. Su propuesta es que los objetos encarnan la experiencia humana que implicó su materialización en el mundo. Dicha experiencia es reinterpretada en la experiencia interactiva de otros agentes gracias al animismo vehicular: debido a la singularidad que evocan cuando se los percibe. Más centrado en una agenda de la antropología del arte, Gell (1998) reconoce la singularidad de la experiencia, pero anteponiendo la agencia en los objetos y su animismo vehicular, y no tanto en los agentes y sus propias subjetividades. Si bien esta propuesta centra la agencia en una situación singular, el único objetivismo que inmiscuye es el del objeto mismo y su agencia y no el del cuerpo de los agentes en la interacción.

Es por esto que la apuesta pragmatista de Joas (2013) es la más acertada para hacer una lectura de la agencia desde la corporalidad del agente en la situación (situación que no siempre es dominante, adversa, etc.). De modo que una acción es creativa, según Joas (2013), en tanto se logra encontrar una solución a un problema tomando como referencia reconstrucciones, esto es, experiencias pasadas, en el contexto de una situación específica, una lógica de corporeidad y de socialización primaria. En sus palabras: «la creatividad se ve como un logro que se produce en situaciones que reclaman una solución (con los medios disponibles), y no necesariamente como la producción incondicionada de algo nuevo» (Joas, 2013: 185). En el contexto de la reintegración, sería la capacidad de un reintegrado de resolver un problema cotidiano

3. Otros autores, como Gramsci (20O3)), abordaron el problema de la resistencia partiendo de la noción de hegemonía como la reproducción de la estructura de las relaciones sociales entre las clases dominantes y las dominadas a partir del uso de la fuerza y la ideología. 
que se le presente con lo que tenga «a la mano»: por ejemplo, saber sobrellevar el estigma con la comunidad o poder desempeñar una labor específica en el trabajo.

Joas (2013), para llegar a ese enunciado, parte del pragmatismo americano, en donde la mirada reflexiva está puesta en la inteligencia y en la reconstrucción como fundamentos de la acción. La inteligencia es la capacidad de resolver problemas que reclaman solución y la reconstrucción es volver a apelar a una experiencia pasada exitosa de resolución de problemas como fundamento para resolver los futuros inconvenientes: aunque no de forma literal, porque las situaciones varían de vez en vez. Es por esto que el autor se distancia de otros enfoques teóricos de la acción: la perspectiva racional y el dualismo cartesiano.

Así, para descartar cercanías reflexivas con respecto a la acción racional, el autor sostiene que ni los fines ni los medios definen la acción de los individuos, y tampoco las acciones cambian según los medios. Para Joas (2013) este esquema denominado «medios-fines», que no es más que el punto de partida de la acción racional, parte de tres supuestos: 1) que el actor es capaz de apelar a una acción dirigida a fines; 2) que el actor domina su cuerpo; y 3) que el actor es autónomo respecto a otros actores y a su entorno. En ese sentido, en contraposición a lo anterior, lo que propone es que los fines no son fijos y, por ende, no determinan la acción: por el contrario, los fines se van transformando en la medida en que el actor encuentra la mejor posibilidad de medios para la resolución de problema. Es la disponibilidad de medios lo que determina los fines: no lo contrario. Por todo esto, para el autor, el esquema medio-fin en sí mismo es lo que impide aclarar los supuestos para el establecimiento de fines y para la acción orientada a fines, pues dicho esquema los trata como evidentes.

En este orden de ideas, los medios tampoco definen la acción, ni tampoco la acción varía según los medios, como lo sostiene este esquema teleológico. La acción, más bien, desde el pragmatismo, es el resultado de la consecución de tres aspectos neurálgicos: la situación, la corporeidad y la socialización primaria, como lo mencioné más arriba. Es decir, que para superar el esquema propuesto por la acción racional hay que reconocer que todo establecimiento de fines viene precedido por la mediación práctica del ser humano y sus situaciones, su cuerpo y el proceso donde se construyen sus esquemas valorativos conocido como socialización primaria. Así mismo, no se debe concebir la percepción o el conocimiento como anteriores a la acción sino más bien como una fase de la acción por medio de la cual ésta se dirige en los contextos situacionales específicos. En pocas palabras, la acción tiene lugar en una situación donde la corporeidad se establece como un elemento constitutivo de la creatividad, no solo en la percepción sino también en la acción en sí, donde detrás de todo conocimiento y valoración hay un antecedente, que es precisamente la socialización primaria. Por ende, el conocimiento en la acción sería más bien el 
resultado de la reflexión y la prerreflexión que opera en la acción, situándose en nuestro cuerpo (Joas, 2013).

El dualismo cartesiano también se descarta en la teoría de la acción creativa. Descartes sostiene que los seres humanos están compuestos por dos sustancias: cuerpo y alma (lo que en la actualidad conoceríamos como mente). Asumiendo una postura racionalista, Descartes asume que lo que le proporciona conocimiento para la toma de decisiones al ser humano es su mente, lo que cala en una suerte de libre albedrío al que estarían sujetas las personas gracias a esta división, a este dualismo. Así, pues, la justificación de los dualistas está en que el cuerpo se divide por medio de la eliminación de una pierna o de un brazo, por ejemplo, pero el alma sigue siendo indivisible. Mientras el cuerpo es visto como procreado, el alma aparece como creada en sí misma.

En todo caso, no se justifica fundar una teoría de la acción basándose en dualismos mente-cuerpo o yo-otro, ya que para Joas (2013) se requiere la integración de estos componentes divididos de forma dualista para construir una teoría de la creatividad de la acción. A esta visión dualista y racionalista, pues, hay que oponerse por varias razones. En primer lugar, para el pragmatismo las personas actúan utilizando su cuerpo, como una unidad situada y socializada, motivadas por sus percepciones. Esto implica que nuestra forma de desenvolvernos en el mundo está implicada por expectativas indeterminadas más que con un claro y preciso esquema de análisis, como sostiene Descartes, ya que es más que suficiente con un entendimiento práctico del mundo que nos rodea. En segundo lugar, siguiendo lo anterior, basta con construir una percepción generalizada del mundo, siendo innecesaria su categorización en unidades fácticas específicas.

Por eso, como sostiene el autor, «el trato humano con la realidad es una interacción flexible entre expectativas globales y percepciones globales, mientras no surjan problemas de acción. Cuando sobrevienen los problemas se produce una puntual concreción de expectativas y percepciones, aunque no un completo replanteamiento (de estas) conforme a una precisa hoja de cálculo» (Joas, 2013: 215-216). Finalmente, la separación rotunda con el dualismo cartesiano está dada porque para el pragmatismo el cuerpo admite la renovación de diferentes formas de percepción y acción, de ahí el ejemplo: si no basta con la mirada para ejemplificar un objeto, apelamos al tacto como recurso complementario.

En suma, «la superioridad de la percepción humana frente a las tentativas de su simulación técnica no deriva en una condición 'superior' del espíritu, que resulta inalcanzable para tales programas, sino de que la percepción se fundamenta en la corporeidad del ser humano» (Joas, 2013:216). Por esto es fundamental complementar un abordaje de la reintegración con la teoría de la acción creativa desde el concepto 
de habitus, asumiendo la importancia del cuerpo en la agencia, y de la agencia como dispositivo estructurante de la estructura (de lo institucional).

Ahora bien, sobre habitus, sin lugar a dudas, el referente principal es Bourdieu (2007), quien lo definió en su momento como una estructura estructurante estructurada que se aprehende (inconscientemente) a través del cuerpo, mediante un proceso de familiarización práctica. En palabras de De Fornel (2005), es un esquema, una disposición corporal y subjetiva de saber-hacer por medio de la cual los sujetos perciben su contexto y se pueden desenvolver en él. Bajando un poco la escala de abstracción, me interesa partir de una concepción de habitus más flexible, como disposición del cuerpo-subjetividad (sin caer en dualismos cartesianos) que implica un esquema de aprendizajes, conocimientos, técnicas, representaciones y disciplinas corporales que los reintegrados deben incorporar y aprehender para lograr (re)integrarse a la vida civil.

Bourdieu $(2007,2008)$ discute las implicaciones del debate histórico en ciencias sociales entre el subjetivismo y el objetivismo, menciona cómo el habitus se configura como proceso inconsciente; pero, al igual que Joas (2013), imprime en el cuerpo la responsabilidad última de la acción, como se ve también en la «Teoría de la estructuración» de Giddens (1995). De ese modo no se trataría, como lo quiero proponer, de asumir el cuerpo como proceso objetivizante de la subjetividad (como lo que opera la acción que decide el pensamiento); no se trata, tampoco, de asumir la subjetividad como proceso subjetivizante de la operación objetiva del cuerpo como realidad material (como lo que se reconfigura por la interacción objetiva de lo corporal con la realidad palpable del mundo); no se trata, de hecho, de pensar por separado el cuerpo y la subjetividad como dos realidades distintas separadas por un conector (separación que sigue siendo un dualismo cartesiano contemporáneo y no racionalista). Se trata de asumir el cuerpo-subjetividad, articulados (-), como realidad social en sí misma: inseparable (algunos estudios han propuesto pensar el cuerpo-emociones como realidad social inmanente) (Scribano, 2012).

El cuerpo no determina la subjetividad y viceversa. El cuerpo encarna la subjetividad y la hace palpable; la subjetividad incorpora el cuerpo y lo hace escuchable. La experiencia corpóreo-subjetiva se siente, se observa y se escucha: la experiencia corpóreo-subjetiva es objetivizante-subjetivizante a la vez. El reintegrado actúa, agencia, resuelve, con su habitus incorporado como cuerpo-subjetividad; no con su cuerpo que responde a una operación mecánica de mando de la subjetividad. Las disposiciones, las técnicas, la moral, los valores, de un escenario dado, son incorporadas, aprehendidas y aprendidas por los sujetos (reintegrados) a través de su habitus en la experiencia; no por su subjetividad que las materializa en movimientos del cuerpo o los actos del habla. El sujeto, pues, encarna su habitus en su experiencia situacional corpóreo-subjetiva que reclama solución. 
Esta forma de comprender la reintegración es clave para evaluar profundamente los procesos que se han llevado a cabo en otros momentos con grupos guerrilleros y paramilitares, tanto en desmovilizaciones masivas como en desmovilizaciones individuales o de grupos pequeños. De igual modo, es pertinente porque permitirá abordar el proceso vigente con los integrantes de la ahora denominada Fuerza Alternativa Revolucionaria del Común (FARC). ${ }^{4}$ Sobre todo por la urgencia manifiesta que tendrá reintegrar a sus más de ocho mil integrantes en aspectos laborales, educativos, familiares, comunitarios, etc. Reintegrarlos pensando en la importancia de su agencia creativa para resolver problemas, por tanto, implicará no sólo no limitar su campo de acción a aquellos escenarios rurales que les sean más conocidos per se; ${ }^{5}$ implicará también reconocer las posibilidades que tienen estos sujetos para desenvolverse en contextos urbanos según sus propios sueños, intereses laborales, capacidades, redes familiares, o simplemente porque antes de ingresar al grupo armado tuvieron la experiencia de vivir en una ciudad.

Un análisis desde esta perspectiva, sin embargo, estaría incompleto si no se asume la importancia de incluir en las reflexiones sobre la reintegración las categorías de raza y género como lo propone el informe del CNMH (2014), sobre todo si se considera que la agencia creativa es una entidad inalienable del cuerpo. Las condiciones situacionales de la acción, pues, no son las mismas para un reintegrado que para una reintegrada; no son las mismas para un reintegrado mestizo que para un reintegrado afro o indígena, etc. El estigma y el prejuicio son entidades situacionales fundamentales. De ahí que Vergara (2014) proponga el concepto de cuerpo vaciado, ${ }^{6}$ clave para entender el estigma inmerso en el proceso de reintegración en la vida cotidiana de los reintegrados cuando está inmiscuido el asunto racial: «hablo de cuerpos vaciados, y no de vacíos, porque los cuerpos diferentes a los que estamos acostumbrados, o a los que conocemos, no están desprovistos de contenido per se» (2014:345). Es decir

4. Es pertinente, además, pensando en el eventual proceso de paz con el ELN.

5. Esto es neurálgico porque en el acuerdo se ha contemplado que la reintegración laboral de estos sujetos debería centrarse en la creación de unidades productivas rurales considerando las ventajas que tienen por su vida rural.

6. Una propuesta que vale la pena mencionar -a propósito de la preocupación por el cuerpo como unidad entrañable de estigmas- es la categoría de cuerpos quebrados («broken bodies» en inglés) de Holmes (2013). A través de un análisis profundo de la vida cotidiana y el dolor de los migrantes mexicanos en Estados Unidos, Holmes (2013), que parte de la antropología del sufrimiento de Farmer (1997), señala que en contextos de profunda desigualdad, racismo, y poco acceso a la salud, los cuerpos encarnan el sufrimiento: lo evidencian en su piel, lo gestualizan, lo tienen incorporado en sus movimientos. Un cuerpo quebrado, sin embargo, no tiene muchas opciones de agenciar ni de ser creativo, pues está sujeto a contextos sumamente adversos. 
que en la interacción con un otro-que se considera diferente por su raza-es vaciado de su trayectoria, de su biografía, del contenido que trae incorporado.

Los imaginarios sociales llenos de estigmas y prejuicios sobre los reintegrados, por su incursión en los grupos armados sumada a las condiciones sociales de exclusión previas, que pudieron haber sido su motivación para ingresar, los ha desprovisto de historia, conocimientos, capacidades y significados: sus cuerpos son vaciados, enfrentan más estigmas que un ciudadano en el contexto de la legalidad. Los prejuicios y estereotipos, entendidos como sentimientos y disposiciones generalmente desfavorables hacia un individuo o un grupo social y que están fundados en el paradigma de la diferencia, tienen influencia nociva en diferentes escenarios de la vida, razón por la cual deben ser tenidos en cuenta en las investigaciones sobre el tema en cuestión. Según Vergara (2014), a partir de dichos prejuicios y estereotipos se construye una narrativa, una historia «oficial», incluso como discurso nacionalista, que refuerza la violencia simbólica estructural y las desigualdades sociales y económicas. En resumen, es crucial comprender el porqué del cuerpo vaciado en la experiencia cotidiana del estigma para investigar las implicaciones sociales de los prejuicios y estereotipos ligados a asuntos raciales entre los reintegrados, que inciden en la configuración de su agencia para resolver problemas puntuales. Un cuerpo vaciado, pues, busca llenarse: busca hacer justicia a un relato, agencia una narrativa lejos de los prejuicios, aunque esto no siempre sea fácil. Por eso es clave entender las condiciones situacionales a las que se enfrenta el agente que ha sido desprovisto de historia.

Varios aportes teóricos similares, y también metodológicos, se sustraen del trabajo de Farnsworth-Alvear (2000), Dulcinea in the Factory, en este caso, ligados a un enfoque de género, para incorporar a un abordaje de la reintegración. Por un lado, esta autora cuestiona exitosamente la categoría de clase, normalmente asumida a priori, al proponer que su comprensión debe estar alineada con el concepto de género entendido en la estructura, no por fuera de ella. Superando el esencialismo intrínseco en la categoría de género, da lugar a una explicación social e histórica para, a su vez, trascender la visión binaria y hegemónica del «hombre-mujer». Al reconocer esto, la autora da lugar a la agencia de los sujetos en la construcción de sus experiencias e interacciones de vida. La postura estructural-funcionalista, evidente en la literatura sobre reintegración en Colombia, se queda corta teóricamente porque niega la agencia y la subjetividad en la experiencia cotidiana de los sujetos de interés. Sin lugar a dudas, un enfoque alternativo para investigar la reintegración debe incluir también una perspectiva de género que supere las visiones homogéneas de la literatura existente. 
Otro de los grandes aportes de Farnsworth-Alvear (2000) para pensar el tema de la reintegración, es metodológico por su novedosa manera de recolectar y analizar la información para reconstruir los relatos, las narrativas y las experiencias; en pocas palabras, para reconstruir la forma como obra la agencia (y por qué no, desde el enfoque de la acción creativa). La autora apeló a una revisión de prensa regional y nacional, de las hojas de vida de las obreras, de las publicaciones de la Oficina de Inspección de Trabajo, de las actas del patronato de obreras, y realizó múltiples entrevistas a los y las diferentes agentes que interactuaron en el contexto de estudio. Darle un rostro humano a la experiencia a través de la fuente oral -más allá de la revisión de los discursos institucionales recurrentes en marcos jurídicos o cifras estadísticas- es una apuesta novedosa que invita al campo de la reintegración a debatir sobre la pertinencia de dar viva voz a los y las implicadas en el programa.

Si una investigación sobre la reintegración se va a centrar en la forma en que se comporta la agencia de los implicados en contextos adversos, la propuesta etnográfica de Bourgois (2010), por mencionar un ejemplo, es sumamente valiosa. Este autor investigó el papel de la disciplina en contextos laborales ilegales de microtráfico (economía subterránea, como la llama). Superando la visión estructuralista de la política antidrogas, este autor concentró su mirada en la agencia cotidiana de los vendedores de droga en Harlem como respuesta a múltiples situaciones adversas: violencia, enfermedades, machismo, pobreza, etc. Metodológicamente, Bourgois (2010) reconoce la agencia de sus interlocutores gracias a su inmersión en el campo con mirada etnográfica: diálogos, entrevistas, observaciones, convivencia, etc. Reconoció, así mismo, cómo la forma en que sus interlocutores asumen singularmente la raza, influye en la toma de decisiones y en la forma de afrontar día a día las adversidades: la supervivencia. Reconocer la agencia, metodológicamente hablando, demandará superar las visiones estructurales para darle un lugar a las experiencias de los implicados a través de apuestas cualitativas, desde sus propias narrativas discursivas y corporales: entrevistarlos y observarlos resolviendo problemas en sus escenarios situacionales laborales, familiares, cotidianos.

\section{Conclusiones}

En síntesis, sustraigo que, teóricamente hablando, hay que destacar los importantes avances en la conceptualización de categorías centrales como desarme, desmovilización, reintegración, reinserción y reconciliación. No hay dudas de que hay una comprensión profunda con respecto a las definiciones, diferencias e implicaciones 
de cada uno de esos conceptos. Sin embargo, así como en la práctica el proceso se ha llevado a cabo de forma homogénea, en el campo investigativo en Colombia se ha entendido cada una de estas categorías de forma análoga con respecto a las demarcaciones institucionales globales, el mandato ONU, y las fronteras casi invisibilizadas en la forma de concebir a los grupos armados y la aplicabilidad del DDR en cada uno de ellos. Esto obedece a que la agenda teórica del DDR en Colombia sigue estando fuertemente supeditada a la revisión de la documentación jurídica que se ha desarrollado sobre dicha temática. Es decir, hay una apuesta y una mirada estructural-funcionalista en la forma de conceptualizar, comprender y abordar la reintegración en Colombia, que ha dejado erróneamente por fuera de la discusión a los implicados y a sus experiencias singulares: sin considerar, además, la incidencia del género, la raza y las identidades regionales o locales, por poner algunos ejemplos.

Otro aspecto teórico problemático que subyace a esta revisión de la literatura tiene que ver con las dimensiones de análisis utilizadas normalmente para investigar el tema de la reintegración en Colombia. Como se puede observar, no hay muchas variantes teóricas para hablar de reintegración más allá de la categoría de reintegración misma, o los conceptos de desarme y desmovilización que hacen parte de la misma política. Algunas revisiones teóricas proponen las diferencias entre reintegración y reinserción, o incluyen en el análisis la categoría de reparación: lo que deja ver cómo la reintegración sigue siendo pensada y abordada únicamente en un contexto de conflicto. Otras pocas propuestas que cabe destacar asumen el papel de la agencia en el proceso y proponen comprenderlo a través de la subjetividad de los implicados y los estigmas hacia los cuales se ven enfrentados según su género. De todos modos, es limitado el estado de la cuestión en cuanto a la comprensión de las dimensiones mismas que propone el programa de reintegración: no hay variados estudios con respecto al asunto de la seguridad de los reintegrados, su vinculación al sistema de salud, al sistema educativo, a la esfera productiva legal, etc.

Por eso no es extraño que, metodológicamente, el actor principal en esta contienda se siga observando desde un plano colectivo. Hay, entonces, una mirada colectivizada que pasa por una preocupación de la academia por el asunto político: la mirada se limita muchas veces a la configuración de partido político resultante de unos acuerdos de paz o una desmovilización masiva, por ejemplo. Este punto supone dos limitaciones más en el estado de la cuestión sobre la reintegración en Colombia. Por un lado, la colectivización del actor ha comprometido los estudios netamente a revisiones documentales y algunos otros a metodologías cuantitativas. La mirada a partir de abordajes más cualitativos de la experiencia del desmovilizado en su agencia, en ese sentido, ha quedado relegada: a excepción de algunos que han apelado a las entrevistas. Por otro lado, se ha caído inevitablemente en una «inocencia 
epistemológica», porque en muchos estudios se afirma comprender el proceso de la reintegración solo abordando la coyuntura institucionalista, sin incorporar la voz y la experiencia de los directamente implicados en las investigaciones.

En Colombia las investigaciones se han concentrado en los marcos jurídicos, estudios institucionalistas y comparativos del proceso desde una agenda estructural-funcionalista, a partir de la coyuntura que implicó la creación de la ACR en esta década: cómo era el proceso antes y cómo es ahora. A nivel local, con fuerte incidencia de lo que acontece a nivel internacional, el DDR se asume como una política global y homogénea que no tiene en cuenta los aspectos singulares de los reintegrados (género, raza, entre otros) pues responde solamente a los nuevos discursos de construcción de Estado y paz con base en asuntos como la gobernanza y los equilibrios políticos. De todos modos, dado que el DDR es un discurso institucionalizado recientemente (años 90), es comprensible que los estudios del campo se encuentren todavía en una fase de discusión jurídico-política, es decir, cómo integrar y legitimar una política global a los casos particulares de conflicto interno en países como Colombia. Las otras dimensiones de la reintegración mencionadas son, por estas razones, aún más limitadas y se circunscriben particularmente al asunto político de la colectividad.

De ahí que una mirada a la agencia del reintegrado en su vida cotidiana, en las diversas esferas en las que se desenvuelve, sea clave para comprender las múltiples formas en que estos sujetos interpretan, reinterpretan y actúan frente al programa de reintegración. Particularmente, el enfoque pragmatista de la teoría de la acción creativa de Joas (2013) puede dar luces en ese sentido al reconocer no solo la importancia de los aspectos institucionales (los marcos jurídicos, por ejemplo) sino también la agencia de los reintegrados, desde sus cuerpos, para resolver problemas cotidianos-situados sin necesariamente responder al dictamen estructural de la institución. Esto por cuenta de la consideración de la estructura estructurante-habitus- de conocimientos que traen los sujetos de su experiencia pasada y que son neurálgicos en su proceso de reintegración en el contexto de una situación específica, una lógica de corporeidad y de socialización primaria. Por eso es necesario que las nuevas miradas sobre el tema den un lugar privilegiado al cuerpo y articulen también la incidencia de la raza y el género (y otras variables) en la configuración de esa agencia.

Metodológicamente, esto implicará también nuevos retos, pues dar cuenta de los procesos cotidianos de la mano de la agencia de los sujetos de estudio debe asumir una mirada más precisa que vaya más allá de la caracterización estadística colectiva e incluya la experiencia individual en el relato, la observación y la participación. Esta mirada teórica-metodológica abre nuevas posibilidades a propósito de otros campos de estudio que busquen reflexionar en torno al cambio social, transiciones, migraciones, desplazamientos y procesos de adaptación de poblaciones diversas 
a nuevos contextos urbanos, rurales, internacionales o de cualquier otra escala geográfica. Pensando en la reintegración masiva de la FARC como resultado del proceso de paz (y una eventual del ELN), la desmovilización de pandillas urbanas, desplazados por el conflicto que llegan a las ciudades, ciudadanos venezolanos o de otras nacionalidades en Colombia, entre otras poblaciones de interés para la intervención del Estado, la mirada situada hacia su agencia cotidiana será fundamental para superar las perspectivas estructural-funcionalistas y así darle un rostro humano a los diversos procesos sociales contemporáneos.

\section{Referencias}

ANDERLINI, S. y CONAWAY, C. (2006). Desarme, desmovilización y reintegración. Recuperado de http://www.reintegracion.gov.co/alta_consejeria/pdf_normatividad/sobre/Desarme_desmovilizacion.pdf

AJAYI, A. y ADESOTE, A. (2013). The Gains and Pains of the Amnesty Programme in the Niger Delta Region of Nigeria, 2007-2012: A Preliminary Assessment. Journal of Asian and African Studies, 48 (4), 506-520.

BOURDIEU, P. (2007). El sentido práctico. Buenos Aires: Siglo XXI editores.

BOURDIEU, P. (2008). Objetivación participante. Boletín oficial del Instituto Nacional de Antropología e Historia, (83-84), 87-101.

BOURGOIS, P. (2010). En busca de respeto. Vendiendo crack en Harlem. México: Siglo XXI editores.

CARRANZA, F. (2012). La reintegración desde el municipio: el rol de las alcaldías de Bogotá y Medellín en la atención a excombatientes. Revista Ópera, (12),159-179.

CHAMORRO, S. (2015). Acercamiento al proceso de Desarme, Desmovilización y Reinserción (DDR) en Nicaragua después de 28 años de la firma de los acuerdos de Esquipulas II. Cultura de Paz, 21 (65), 15-27.

CORREA, N. (2007). Reinserción y reparación. Vniversitas, (114), 251-289.

COURSON, E. (2011). Political Marginalization, Repression, and Petro-Insurgency in the Niger Delta. African Security, 4 (1), 20-43.

DAVIDHEISER, M. y NYIAYAANA, K. (2011). Demobilization or Remobilization? The Amnesty Program and the Search for Peace in the Niger Delta. African Security, 4 (1), 44-64.

DE FORNEL, M. (2005). Habitus y etnométodos. En P. Encrevé y R. Lagrave (Comp.), Trabajar con Bourdieu (pp. 235-244). Bogotá: Universidad Externado de Colombia. 
FARMER, P. (1997). On suffering and Structural Violence: a view from below. Berkeley: University of California Press.

FARNSWORTH-ALVEAR, A. (200o). Dulcinea in the Factory: Myths, Morals, Men, and Women in Colombia's Industrial Experiment, 1905-1960. Estados Unidos: Duke University Press.

GELL, A. (1998). Art and Agency: An Anthropological Theory. Oxford: Clarendon.

GIDDENS, A. (1995). La constitución de la sociedad: bases para la teoría de la estructuración. Buenos Aires: Amorrortu Editores.

GIRALDO, S. (2010). Contextualización teórica e histórica de la reintegración social y económica de desmovilizados en Colombia. En Poliantea, 35-52.

GIRALDO, J.y MESA, J. (2013). Reintegración sin desmovilización: el caso de las milicias populares de Medellín. Colombia Internacional, 77 (1), 217-239.

GLEICHMAN, C., ONDENWALD, M., STEENKEN, K. y WILKINSON, A. (2004). Desarme, desmovilización y reintegración. Guía teórica y práctica. Frankfurt: Imprenta Hassmuller.

GOFFMAN, E. (2004). La presentación de la persona en la vida cotidiana. Buenos Aires: Amorrortu.

GÓMEZ, A. (2007). Mercado de trabajo y paz en Colombia; una lectura sobre el proceso de desmovilización de actores armados ilegales. Revista facultad de trabajo social, (23), 89-99.

GRAMSCI, A. (2003). Cartas de la cárcel. México: Ediciones Era.

HOLMES, S. (2013). Fresh fruit, broken bodies. Migrant farmworkers in the United States. Berkeley. Estados Unidos: University of California Press.

HUMPHREYS, M., y WEINSTEIN, J. (2007). Demobilization and Reintegration. Journal of Conflict Resolution, (51), 531-567.

HUNDEK, L. (2016). Mujeres reinsertadas: postconflicto en Barranquilla. Advocatus, 14 (27), 65 - 82.

JOAS, H. (2013). La creatividad de la acción. Madrid: Centro de Investigaciones Sociológicas.

LABRADOR, K. y GÓMEZ, M. (2010). Desarme, Desmovilización y Reintegración en Colombia. Bogotá: Fundación Antonio Restrepo Barco.

LUNA, M. (2007). El reconocimiento de sí mismo en los militantes del M-19. Revista Sociedady Economía, (13), 44-65.

MESA, J.D. (2015). La Población en la mitad: reflexiones sobre Bojayá y La Sierra. Trans-pasando Fronteras, (8), 125-143.

NUSSIO, E. (2012). La vida después de la desmovilización. Bogotá: Universidad de los Andes.

NUSSIO, E. (2013). Desarme, desmovilización y reintegración de excombatientes: políticas y actores del postconflicto. Colombia Internacional, 77 (1), 9-16. 
PÉREZ, W. (2005). Orden jurídico, negociación, paz y reinserción. La constante imbricación entre guerra, política y derecho en Colombia. Estudios Políticos, (27), 67-10o.

ROMERO, T., RESTREPO, N. y DÍAZ, I. (2009). Factores psicosociales que inciden en la reintegración social de tres reclusos con vínculos a los grupos armados ilegales (FARC-EP, UC-ELN y AUC) del Centro Penitenciario y Carcelario de Villahermosa. Pensamiento Psicológico, 6 (13), 219-238.

SCOTT, J. (1976). The moral economy of the peasant: Rebellion and subsistance in Southeast Asia. New Haven: Yale University Press.

SCOTT, J. (2008). Weapons of the Weak: Everyday Forms of Peasant Resistance. New Haven: Yale University Press.

SCRIBANO, A. (2012). Sociología del cuerpo-emociones. Revista Latinoamericana de Estudios sobre Cuerpos, Emociones y Sociedad, 4 (10), 91-111.

TAMAYO, C., RESTREPO, N. y GUTIÉRREZ, M. (2012). Diagnóstico del proceso educativo en salud de la población desmovilizada del municipio de Medellín, Antioquia 2011. Revista CES Salud Pública, 3 (2), 141-151.

THOMPSON, E.P. (200o). Costumbres en común. Barcelona: Editorial Crítica.

THOMPSON, E.P. (2014). La economía moral de la multitud y otros ensayos. Bogotá: Ediciones desde abajo.

VERGARA, A. (2014). Cuerpos y Territorios vaciados: ¿en qué consiste el paradigma de la diferencia? ¿Cómo pensamos la diferencia? Revista CS, (13), 338-360.

VILLARRAGA, Á. (2013). Experiencias históricas recientes de reintegración de excombatientes en Colombia. Colombia Internacional, (77), 107-140.

VILLARRAGA, Á. (2015a). Desmovilización y reintegración paramilitar. Panorama posacuerdos con las AUC. Bogotá: CNMH.

VILLARRAGA, Á. (2015b). Rearmados y reintegrados. Panorama posacuerdos con las AUC. Bogotá: CNMH.

ZIRION, I. (2012). Los procesos de desarme, desmovilización y reintegración de excombatientes desde la perspectiva de género. Cuadernos de trabajo, (58), 2-46. 


\section{Documentos emitidos por organismos}

AGENCIA COLOMBIANA PARA LA REINTEGRACIÓN. (2016). La Ruta de la reintegración. Bogotá: ACR.

CENTRO NACIONAL DE MEMORIA HISTÓRICA. (2014). Desafiospara la reintegración:enfoques de género, edad y etnia. Bogotá: CNMH.

ESCUELA DE CULTURA DE PAZ. (2011). Introducción al Desarme, Desmovilizacióny Reintegración (DDR) de excombatientes. Barcelona: ECP.

ORGANIZACIÓN DE LAS NACIONES UNIDAS. (2006). Disarmament, Demobilization and Reintegration. Recuperado de http://www.unddr.org/iddrs/o1/20.php

\section{Fuentes jurídicas}

ALTO COMISIONADO PARA LA PAZ. (noviembre de 2016). Acuerdo final para la terminación del conflicto y la construcción de una paz estable y duradera. Recuperado de http://www. altocomisionadoparalapaz.gov.co/procesos-y-conversaciones/Documentos\%2ocompartidos/24-11-2016NuevoAcuerdoFinal.pdf

CONGRESO DE COLOMBIA. (25 de julio de 2005). Por la cual se dictan disposiciones para la reincorporación de miembros de grupos armados organizados al margen de la ley, que contribuyan de manera efectiva a la consecución de la paz nacional y se dictan otras disposiciones para acuerdos humanitarios. [Ley 975 de 2005]. Recuperado de http://www.fiscalia.gov.co/jyp/ wp-content/uploads/2013/o4/Ley-975-del-25-de-julio-de-2005-concordada-con-decretos-y sentencias-de-constitucionalidad.pdf

DEPARTAMENTO NACIONAL DE PLANEACIÓN. (1 de diciembre de 2008). Política nacional de reintegración social y económica para personas y grupos armados ilegales. [CONPES 3554 de 2008]. Recuperado de http://www.reintegracion.gov.co/es/la-reintegracion/centro-de documentacion/Documentos/Documento\%20Conpes\%203554\%20l\%2OPol\%C3\%ADtica\%2onacional\%2ode\%2oreintegraci\%C3\%B3n\%2osocial\%2Oy\%2oecon\%C3\%B3mica\%2opara\%20 personas\%20y\%2ogrupos\%2oarmados\%2oilegales.pdf

PRESIDENCIA DE LA REPÚBLICA. (3 de noviembre de 2011). Por el cual se crea la Agencia Colombiana para la Reintegración de Personas y Grupos Alzados en Armas y se establecen sus objetivos y estructura. [Decreto 4138 de 2011]. Recuperado de http://wsp.presidencia.gov.co/Normativa/ Decretos/2011/Documents/Noviembre/o3/dec413803112011.pdf

PRESIDENCIA DE LA REPÚBLICA. (29 de mayo de 2017). Por el cual se modifica la estructura de la Agencia Colombiana para la Reintegración de Personas y Grupos Alzados en Armas y se dictan otras disposiciones. [Decreto 897 de 2017]. Recuperado de http://es.presidencia.gov.co/normativa/normativa/DECRETO\%20897\%2oDEL\%2O29\%20DE\%2OMAYO\%2ODE\%2O2017.pdf 